\title{
Implementation of a decentralized real-time management system for electrical distribution networks using the internet of things in smart grids
}

\author{
Anass Lekbich, Abdelaziz Belfqih, Chaimae Zedak, Jamal Boukherouaa, F. Elmariami \\ Energy and Electrical Systems laboratory, National Higher School of Electricity and Mechanics (ENSEM), Hassan II \\ University, Morocco
}

\begin{tabular}{|c|c|}
\hline Article Info & ABSTRACT \\
\hline Article history: & \multirow{10}{*}{$\begin{array}{l}\text { Intelligent management of the electrical network is the implementation of an } \\
\text { integrated system based on a reliable and secure communication architecture } \\
\text { for transmitting end-to-end information between the equipment and the } \\
\text { management system. The main objective of this work is to develop an } \\
\text { intelligent telecontrol solution for the electrical distribution network } \\
\text { combining communication techniques and an intelligent reconfiguration } \\
\text { strategy. The solution is based on a graphic model and a secure } \\
\text { communication architecture using the internet of things to ensure flexibility } \\
\text { in terms of management of the intelligent network. This intelligent multi- } \\
\text { criteria solution uses a secure communication architecture and the MQTT } \\
\text { protocol to ensure system interoperability and security. The tests were carried } \\
\text { out on the IEEE } 33 \text { bus network and consequently, an optimization of the } \\
\text { losses and a clear improvement in the nodal voltage were recorded despite } \\
\text { the variation of the electric charge. }\end{array}$} \\
\hline Received Mar 31, 2020 & \\
\hline Revised Jun 16, 2020 & \\
\hline Accepted Apr 24, 2021 & \\
\hline Keywords: & \\
\hline Distribution networks & \\
\hline Internet of things & \\
\hline Power optimization & \\
\hline Reconfiguration & \\
\hline Remote control & \\
\hline
\end{tabular}

SCADA system

Smart grid

TOPSIS

This is an open access article under the CC BY-SA license.

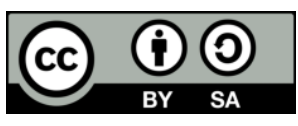

\section{Corresponding Author:}

Anass Lekbich

Energy and Electrical Systems laboratory, Team RECS

National School of Electricity and Mechanics (ENSEM-Hassan II University of Casablanca, Morocco)

Road El Jadida, Km 7, BP: 8118, Oasis Casablanca, Morocco

Email: anass.lekbich@gmail.com

\section{INTRODUCTION}

- Challenges and motivations

Modern society has faced several problems related to the availability of sustainable and clean energy due to the high demand from industry and the dependence of communication systems and data processing on electric power. In addition, electrical networks, these days, are more and more under the operating constraint and are subject to the phenomenon of degradation. Intelligent control of distribution networks keeps the equipment in normal condition and prolonged its service life, which improves the overall reliability of the system, also reducing power losses, notably created by the voltage variation during the distribution process. These issues prompted energy stakeholders to engage in in-depth discussions on power grids, which identified typical grid problems. Today's electricity networks have to cope with high energy consumption by increasing $\mathrm{CO}_{2}$ emissions [1].

A typical solution to the different challenges of the networks remains complicated especially in the current conditions with the competition between the utilities, the manufacturers and the technologies. The implementation of digitization techniques has rapidly transformed customer requirements and has squeezed the gains of stakeholders in the energy sector. Intelligent power grids are therefore a solution to face the 
major challenges of conventional power systems such as reliability, safety, energy costs and ultimately the environment [2].

These technologies bring together field experiences in a mutated and digitized thinking framework of the entire energy sector to make the network flexible, efficient and intelligent. This makes it possible to implement high-tech equipment (reclosers, circuit breakers, remote-controlled switches, digital relays, digital meters), communication media with minimum response time and high coverage [3] (the M2M solution, the internet of things (IoT)) and intelligent software tools to operate the power grid in the best possible conditions.

In the literature, several works address the topic of smart grids as a high-tech solution to solve problems related to network stability, monitoring, management and automation by applying several techniques and tools. In this article, a decentralized architecture of voltage control based on the IoT is proposed, the solution changes the topology of the network to stabilize the voltage, reduce losses and improve the reliability. In the same concept of decentralization and control, the work [4] proposes a decentralized system in real time for the regulation of hierarchical frequency in the electricity network, which makes it possible to compensate the transactions of producers/consumers in real time. This made the system robust and stable. Other articles consider the network as a multicriterion system to regulate the voltage via optimal reconfiguration. In article [5], a modified heuristic approach for the distribution system was implemented to control the nodal voltage and optimize the reliability of the electrical distribution system by applying the reconfiguration to the system. In the same orientation, the paper [6] introduces a new reliability-based reconfiguration method to improve distribution system performance.

D. L. Duan et al. [7], the network reconfiguration problem for loss reduction and reliability improvement was formulated to be solved using improved GA. For a radial system of 136 buses, the power losses were improved from $293.29 \mathrm{~kW}$ in [8] and $280.19 \mathrm{~kW}$ in [9] to $280.19 \mathrm{~kW}$ for this method in [7] with a time of execution inferior to that of [9] (402.36 s for [9] and $33.98 \mathrm{~s}$ for [7]). Moreover, a comparison between the sizes of the networks has shown that the problem resolution time increases in a very significant way.

In a real network, the size is very important which limits the implementation of these techniques to generate the distribution networks. In this article, the network will be operated under its minimal conditions. In other words, the network structure will be decentralized to work with adjacent subnetworks. In this orientation, a decentralized architecture is a good tools to solve the calculation time issue. Our goal is to develop an intelligent, decentralized and flexible multi-criteria electricity network management solution based on the IoT to control voltage, minimize Joule losses and optimize grid reliability.

\section{THE ADVANCED CONTROL SOLUTIONS ADAPTED TO SMART GRIDS ISSUES}

The smart grid is characterized by the following elements [10]: self-healing, usability, cybersecurity, the ability to optimize energy costs, respectful of nature, the use of robust two-way communications, advanced sensors that are fundamental and widely distributed on the various points of the electrical network to provide a lot of crucial information about the network in real time [11], reliability and security of energy supply and use. These elements require a complex system including digital field equipment, robust routers/gateways, secure communication protocols, intelligent and evolutionary control and management software, and finally a reliable decision-making strategy.

Controlling the SG network in terms of secure and reliable operations involves designing a complex infrastructure [12]. The electrical energy management system includes algorithms for performing various operations, such as defining the operating point of elements of the electrical network [13], controlling the electrical energy and finally regulating the voltage in all bus of the networks [14]. The management of electrical distribution networks is therefore a problem of multicriteria decision-making based on the availability of real-time information of all types of equipment [15]. The complexity of this problem is related to the tree architecture of the network, the unpredictability of faults, the load consumed and the limitation of algorithms to process information and make decisions in real time. So the driving and energy management system must have algorithms that work in parallel for intelligent network management [16].

The reliable application of electrical and communication parameter detection techniques imposes intelligent control and network management strategies. Several types of control strategies have been developed for flexible and intelligent management of distribution networks [17]: centralized [18] and decentralized. In this work decentralized strategie is develloped to manage the ditribution networks in reel time. 


\section{PROBLEM FORMULATION OF DECENTRALIZED RECONFIGURATION OF DISTRIBUTION NETWORKS}

To perform a decentralized electrical network reconfiguration solution, the problem of controlling the architecture of the electrical network is divided locally into several independent zones, see Figure 1. We used the graph to model the link between the network area and the consumption point, which will allow us to elaborate a matimatical equation to evaluate power flow, voltage, and electrical architecture.

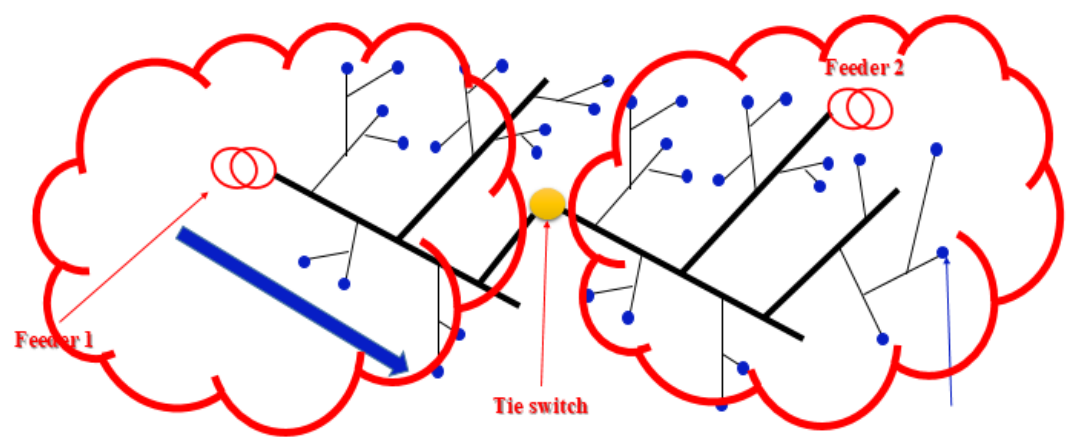

Figure 1. The decomposition of the electrical distribution network

\subsection{The mathematical model of the decentralized control of smart grids}

$\mathrm{G}$ is the graph that represents the radial electrical distribution network composed of $\mathrm{N}$ bus and $\mathrm{L}$ branches. We take the voltage at the source as a reference during this study. The adjacent matrix A of the network is defined as follows:

$a i j=1$ if there is a link between the point $\mathrm{i}$ and $\mathrm{j}$

aij $=0$ if not

The Figure 2 shows a single line diagram of an electrical distribution line.

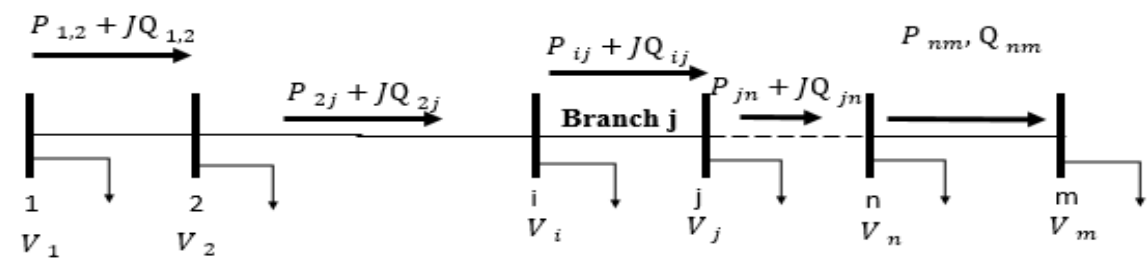

Figure 2. The power flow in the electrical link

$V_{i j}=V_{i}-V_{j}$ is the branch voltage $i j$

w named the voltage column is used for simplification as follows:

$$
w i=V_{0}-V_{i}=1 p u-V_{i}
$$

$I_{i}$ is the electric current absorbed by the load in point of consumption i and Iij the current which passes on the branch i. $\mathrm{Z}$ is the diagonal matrix of branch impedances:

The relation between the current and the voltage is determined using the Kirchhoff laws which allows to formalize the voltage by the following formula [19-20]:

$$
\mathrm{Vij}=\mathrm{Vi}-\mathrm{Vj}
$$

The equation $w=A^{*} \cdot Z \cdot A^{* T} \cdot I$ expresses the relation between the consumed current, the line current, the branch voltage and the nodal voltage in order to calculate the voltage at each bus, the joule losses during the energy distribution and the electrical flux [21].

Where $A^{*}$ is the transitive closure of the graph G [22]. The voltages of each node after each configuration are calculated iteratively as follows [23]:

Step1: calculate w 
Step 2: calculate the injected current for each bus for the kth iteration using:

$$
I_{i}^{k}=\left(\frac{P_{i}+j Q_{i}}{V_{i}^{k}}\right)^{*}
$$

Step 3: calculate the voltage column for the $(\mathrm{k}+1)^{\text {th }}$ iteration: $w^{k+1}=A^{*} \cdot Z \cdot A^{* T} \cdot I^{k}$

The calculations are repeated up to $\left|w^{k+1}-w^{k}\right|<\varepsilon$

Where $\varepsilon$ is the condition of convergence.

\subsection{The network model after the installation of the switches}

The location of switches in the distribution network must be taken into account by our mathematical model and will be coded as follows:

$$
B=\left(b_{j}\right)_{1 \leq j \leq n} \text { with } b_{j}=0 \text { if there is a switch device in the branch } \mathrm{j} \text { and } b_{j}=1 \text { if not. }
$$

The vector B is defined as being the indicator of location of the breaking devices in an electrical distribution network. This vector plays a very important role in the remote control of the network, it allows the system to calculate and make the decision to detect the points of the electric network which can be modified to change the topology. The parameter s reflects the state of the switch organs:

$S_{i}^{k=} l$ the installed switch in i is closed in time k.

$S_{i}^{k=0} 0$ the installed switch in i is open in time $k$. The parameter s reflects the state of the switch organs:

$S_{i}^{k=1}$ the installed switch in $\mathrm{i}$ is closed in time $\mathrm{k}$.

$S_{i}^{k=} 0$ the installed switch in $\mathrm{i}$ is open in time $k$.

\subsection{Formulation of the optimization problem}

The goals of the smart grid include reducing the power losses, control and voltage regulation in the various consumption points [24], improving reliability and ultimately minimizing operating and maintenance costs. Achieving these goals means the ability of the network's management and monitoring system to generate orders respecting the operating constraints. These orders will be sent remotely via a communication system to the switches which allow conducting the power distribution network. The criteria of choice and accurate decision are detailed as follows:

\section{Minimizing the power loss:}

$$
P_{\text {loss }}=\sum_{\substack{i=1 \\ i \neq j}}^{n} \sum_{j=1}^{n}\left(R_{i j} \cdot\left|I_{i j}\right|^{2}\right)
$$

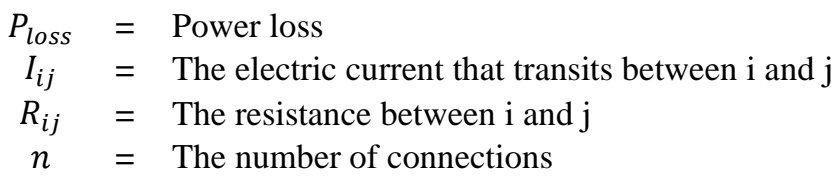

\section{Minimize the number of switching operations (NSW):}

In this article, we propose the NSW function that represents the sum of state change number of switches installed in the power grid.

$$
N S W(x)=\sum_{i=1}^{n}\left(s_{i}^{k}-s_{i}^{k+1}\right)^{2}
$$

\section{Balancing the nodal voltage:}

During the energy distribution process, customer consumption is generally unpredictable; the telecontrol system must generate at every moment the topology and change orders to stabilize the voltage value close to $1 \mathrm{pu}$.

\section{Constraints:}

In the searching process of optimal solution in various optimization problems, many constraints are included. In smart grids, the constraints of operations minimize the decisions made by the operators. Generally, these constraints are related to the nodal voltage and the radial architecture. Each of these constraints are mathematically formalized in the form of the following equations:

$$
0.95 p u<V_{i}<1,05 p u \quad N-L-1=0
$$


where $\mathrm{N}$ : the number of buses in electrical networks and the $\mathrm{L}$ the number of branches.

\subsection{Reconfiguration method}

An algorithm has been proposed in this paper to generate the opening/closing orders of switches see the Figure 3. This algorithm takes into account the number of customers, the electrical parameters, the consumed power and the connection between production and consumption and this in order to respect the requirements of network operation (minimize losses and improve the voltage profile).

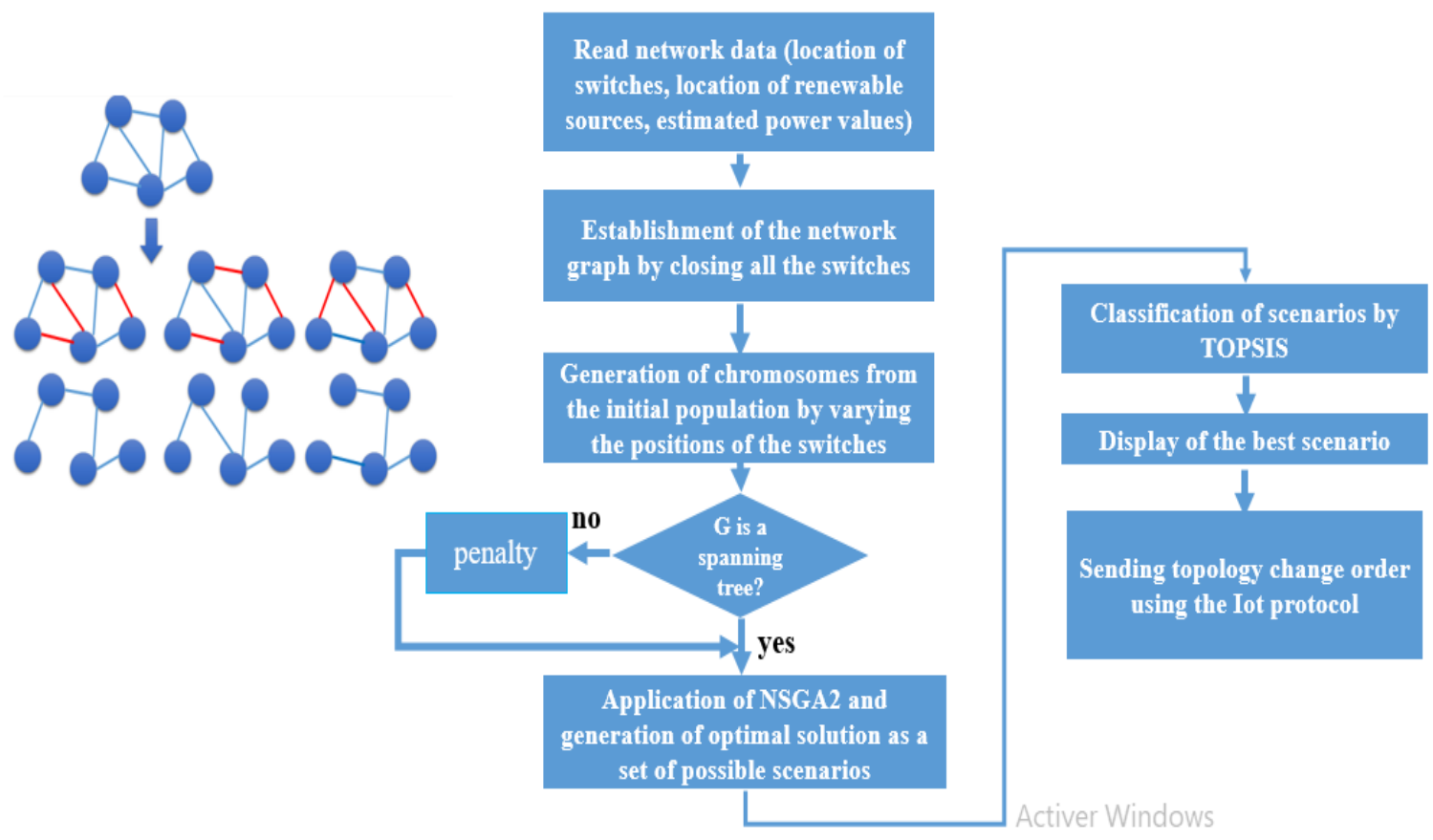

Figure 3. The flow chart of the network control method

\subsection{Multicriteria decision analysis based on the TOPSIS method}

In order to determine the best scenario, we will need a decision analysis method supporting many criteria, with the ability to classify the real electrical network scenarios. The TOPSIS is a simple and good decision analysis method and widely used in optimization problems [25]. It is a powerful decision-making method based on the concept of distance between the scenario and the two positive ideal points (the ideal positive solution, and the ideal negative solution). The motivation to use this technique is that it is simple to program and does not require a lot of calculation, which exactly meets our main objective of manipulating several objectives with the minimum calculation time. The specific steps of TOPSIS method used in this work are detaid in [25] and [23].

Step 1: Establishment of a decision matrix in order to classify all the scenarios. This matrix contains $\mathrm{N}$ scenarios and L decision criteria.

Step 2: In order to have the same dimension of the criteria, a normalization technique is used at this stage by the calculations matrix also called normalized decision matrix.

Step 3: Calculation of the normalized weighted matrix by multiplying the columns of the normalized decision matrix by the associated weights, the sum of the weights must be 1 .

Step 4: Determination of the PIS positive ideal solution and the NIS negative ideal solution.

Step 5: Calculation of the distances of each scenario from the positive ideal solution PIS and the negative ideal solution NIS and classification of all the scenarios.

\section{THE ARCHITECTURE OF THE PROPOSED SOLUTION}

To design an intelligent IoT-based smart grid control and management solution, the proposed architecture must be able to support real-time Internet-based measurement [26] and control capabilities [27] by offering features such as real-time RTU management, intelligent electronic devices (IEDs) configuration, 
a simple and ergonomic human machine application [28], fault management and voltage control, which facilitates the adoption of cloud technologies already available for the management of distribution networks [29] and makes use of the latest data processing and predictive analysis techniques. The Figure 4 illustrates the architecture of the the proposed solution. The architecture consists of 4 essential parts:

a. The first part is the equipment layer, it is the field part that uses many types of equipment to operate electrical networks efficiently. Composed of smart communicating sensors and able to measure the different values of the electrical distribution system, these sensors are connected to the control system by RTU (remote terminal unit). The RTU offers the necessary signal processing capability to be able to identify information from the power grid and adapt it to the communication medium set up to automate and control the power grid [30]. This layer makes it possible to collect and transmit the digital data of the active and reactive powers, the values of the electrical voltage, the current, the presence of fault, the state of the equipment and the electrical networks.

b. The second layer is the communication layer: gathers the gateways, VPN (virtual private network) routers and modems needed to access the communication network used by the power grid management company [31].

c. The third layer is the communication protocol part used to route information between RTU and the SCADA system. The protocol must be secure and interoperable adapted to the needs of connected objects including bandwidth and energy consumption. In this work, we use the MQTT to route information between RTU and the control system.

d. The fourth layer is the most important part of the solution, it is the application layer that manages all equipment in real time. It includes many decision-making tools and the human machine interface to schematize the state of the electrical network. Our reconfiguration program is implemented in this part.

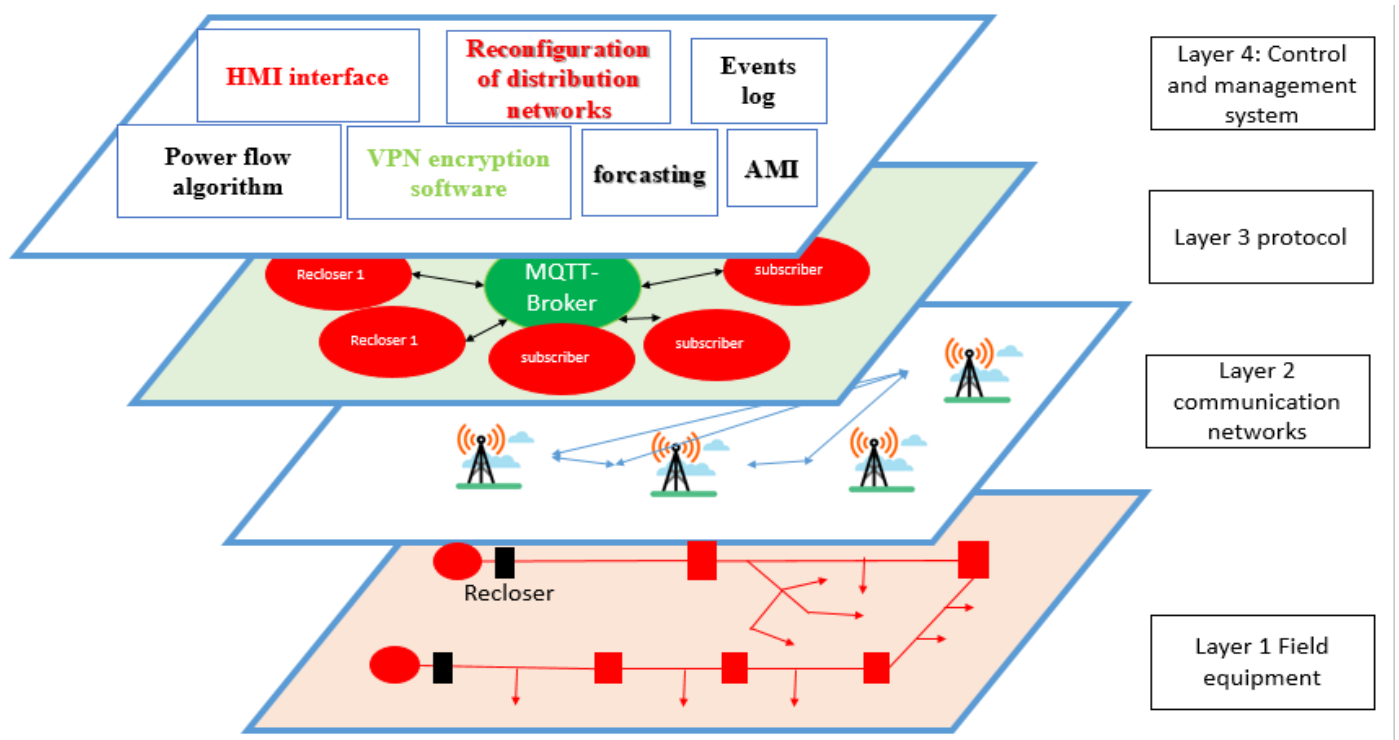

Figure 4. The architecture of distribution management system based on IoT

The proposed communication support, see Figure 5, is based on the public internet ensuring security and interoperability. The communication architecture is decentralized. Data exchange is done via the Internet using the MQTT protocol as a lightweight protocol for publish/subscribe messaging transport based on TCP/IP [32]. The sensors used in this solution are the capacitive voltage capacitors used to give the value of the high-voltage, the current sensor that gives the current value on the electrical line and a Raspberry Pi 3 to store information and to emulate the IEEE 33 BUS. The VPN router gives us the possibility of establishing a secure link using a virtual private protocol tunnel powered by OpenVPN. The OpenVPN application is installed in a remote server ubuntu 18.04. 


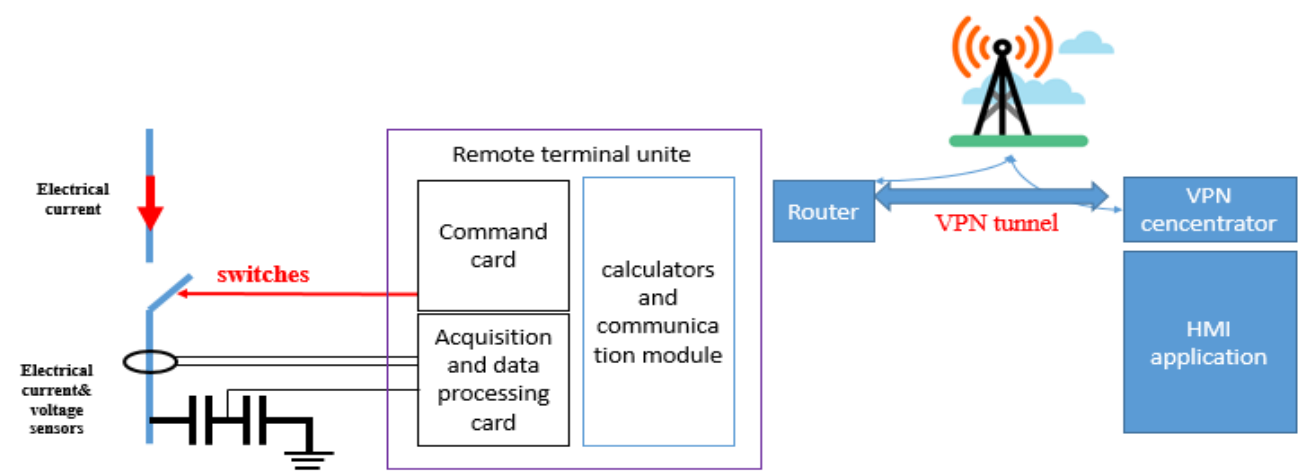

Figure 5. The communication link

\section{SECURITY}

The communication between the SCADA system and the field devices is an encrypted connection according to IEC62351 standard requiring the following points [23]; a) Confidentiality: the confidentiality of communication is implemented by one of the cryptographic techniques allowing to transmit the end-to-end information in the form of encrypted requests and requiring a very important calculation time to be able to decrypt them. These algorithms need to be constantly improved to be on standby and ensure the security of the system; b) Integrity: the information must be protected against modifications; c) Availability: during a request from the SCADA system or the user, the device must be available; d) Authentication: the device must have access permission and the source must be known; e) Non-repudiation: the user does not have the right to refuse to respond to the exchange of information.

\section{IMPLEMENTATION OF THE PROPOSED SOLUTION UNDER PYTHON}

To develop the multicriteria application of control, monitoring and decision, a code under python is produced in this article. The code contains the libraries needed to manipulate the matrix and execute the reference method. These are the essential parts of our solution code; a) To model the electrical network, specific phyton libraries were used to create the graphical interface, see Figure 6. This interface displays information on the electrical network in the form of a block diagram; b) The state of the switches is modeled by red (closed)/green (open) colors; c) Power lines are represented by single-phase lines; d) Specific areas are used to display the electrical values and curves to represent the trend in real time.

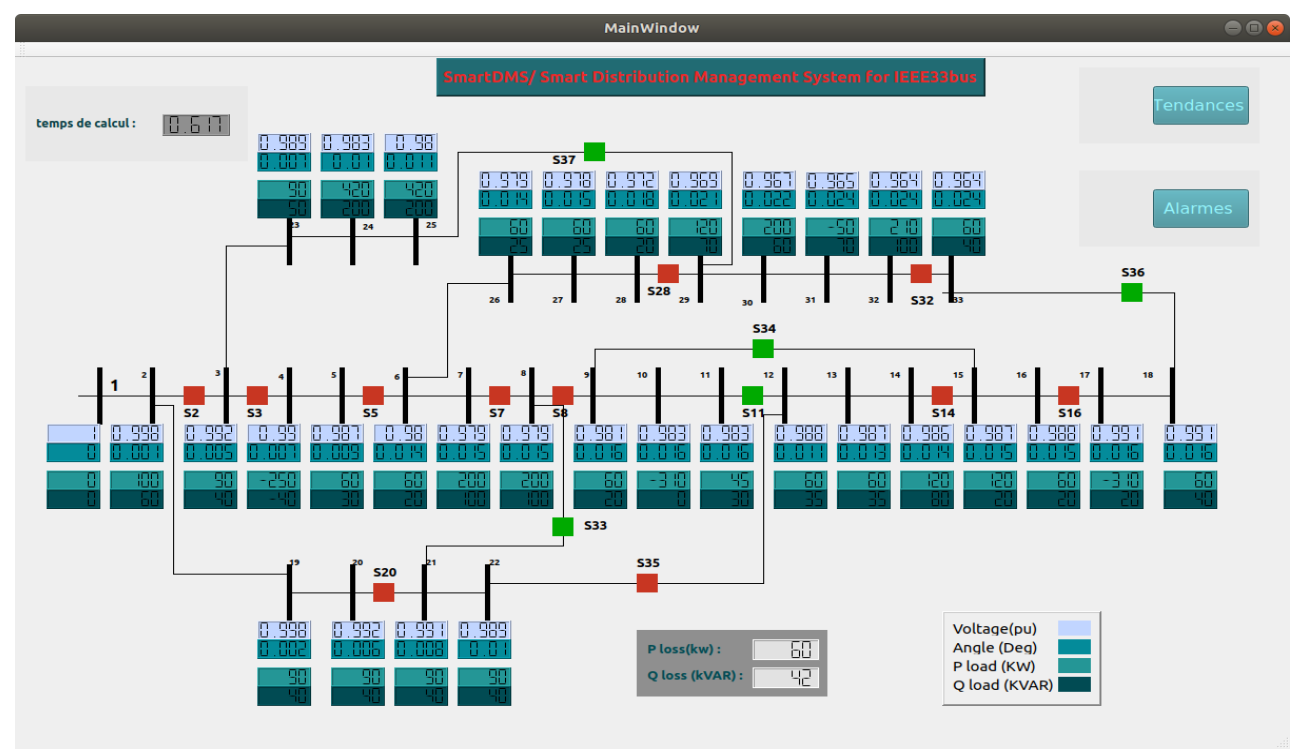

Figure 6. The human machine interface of the IEEE 33 bus network solution 


\section{TEST AND RESULTS}

The network used in this work is the IEEE 33 bus, the standard information is itemized in Table 1. In order to test the solution, several scenarios were created by modifying the consumed power. The change of this power varies the electrical parameters which will create power losses and generate voltage drops. The solution seeks, at each power change, to determine the optimal adapted configuration. The test conditions are represented in Table 2. After performing this test, the solution developed in this paper finds the optimal network configuration. In addition, we plot the voltage profile for all the tests, and we find that our algorithm can find a new configuration that respect the voltage constraints, improves the voltage profile and reduces $\mathrm{P}$ Losses. After analyzing Figures 7 and 8, we notice that the nodal voltage is automatically improved just by changing the toplogy of the electrical network. A solution also obtained by this method stabilizes the voltage during the electrical energy distribution process.

Table 1. IEEE 33 bus network data

\begin{tabular}{|c|c|c|c|c|c|c|c|c|c|}
\hline Bus $N^{\circ}$ & $\mathrm{P}(\mathrm{kW})$ & $\mathrm{Q}$ (kVAR) & $\mathrm{R}(\mathrm{m} \Omega)$ & $\mathrm{X}(\mathrm{m} \Omega)$ & Bus $N^{\circ}$ & $\mathrm{P}(\mathrm{kW})$ & $\mathrm{Q}$ (kVAR) & $\mathrm{R}(\mathrm{m} \Omega)$ & $\mathrm{X}(\mathrm{m} \Omega)$ \\
\hline 2 & 100 & 60 & 0.0922 & 0.0470 & 17 & 60 & 20 & 1.2889 & 1.7210 \\
\hline 3 & 90 & 40 & 0.4930 & 0.2512 & 18 & 90 & 40 & 0.7320 & 0.5739 \\
\hline 4 & 120 & 80 & 0.3661 & 0.1864 & 19 & 90 & 40 & 0.1640 & 0.1565 \\
\hline 5 & 60 & 30 & 0.3811 & 0.1941 & 20 & 90 & 40 & 1.5042 & 1.3555 \\
\hline 6 & 60 & 20 & 0.8190 & 0.7070 & 21 & 90 & 40 & 0.4095 & 0.4784 \\
\hline 7 & 200 & 100 & 0.1872 & 0.6188 & 22 & 90 & 40 & 0.7089 & 0.9373 \\
\hline 8 & 200 & 100 & 0.7115 & 0.2351 & 23 & 90 & 50 & 0.6512 & 0.5084 \\
\hline 9 & 60 & 20 & 1.0299 & 0.7400 & 24 & 420 & 200 & 0.8980 & 0.7091 \\
\hline 10 & 60 & 20 & 1.0440 & 0.7400 & 25 & 420 & 200 & 0.8959 & 0.7071 \\
\hline 11 & 45 & 30 & 0.1967 & 0.0651 & 26 & 60 & 25 & 0.2031 & 0.1034 \\
\hline 12 & 60 & 35 & 0.3744 & 0.1298 & 27 & 60 & 25 & 0.2842 & 0.1447 \\
\hline 13 & 60 & 35 & 1.4680 & 1.1549 & 28 & 60 & 20 & 1.0589 & 0.9338 \\
\hline 14 & 120 & 80 & 0.5416 & 0.7129 & 29 & 120 & 70 & 0.8043 & 0.7006 \\
\hline 15 & 60 & 10 & 0.5909 & 0.5260 & 30 & 200 & 100 & 0.5074 & 0.2585 \\
\hline 16 & 60 & 20 & 0.7462 & 0.5449 & 31 & 150 & 70 & 0.9745 & 0.9629 \\
\hline \multirow[t]{2}{*}{17} & 60 & 20 & 1.2889 & 1.7210 & 32 & 210 & 100 & 0.3105 & 0.3619 \\
\hline & & & & & 33 & 60 & 40 & 0.3411 & 0.5302 \\
\hline
\end{tabular}

Table 2. Results of the different scenarios

\begin{tabular}{|c|c|c|}
\hline Scenarios & $\mathrm{P}$ & Q \\
\hline TEST1 & IEEE33 Bus standard value & IEEE33bus standard value \\
\hline TEST2 & $\begin{array}{l}\text { and } 18 \text { simultaneously with a decrease in the active } \\
\text { power } \mathrm{P} \text { absorbed on buses } 23,24 \text { and } 25 \text {. }\end{array}$ & $\begin{array}{l}17 \text { and } 18 \text { simultaneously with a decrease in the } \\
\text { reactive power absorbed on buses } 23,24 \text { and } 25 \text {. }\end{array}$ \\
\hline & In this test, we increase the power on buses $9,10,17$ & We increase the values of $Q$ on buses $9,10,17$ and \\
\hline TEST3 & $\begin{array}{l}\text { and } 18 \text { simultaneously with a decrease in the active } \\
\text { power } \mathrm{P} \text { absorbed on buses } 7,8,23,24 \text { and } 25 \text {. }\end{array}$ & $\begin{array}{l}18 \text { simultaneously with a decrease in the reactive } \\
\text { power absorbed on buses } 7,8,23,24 \text { and } 25 \text {. }\end{array}$ \\
\hline
\end{tabular}
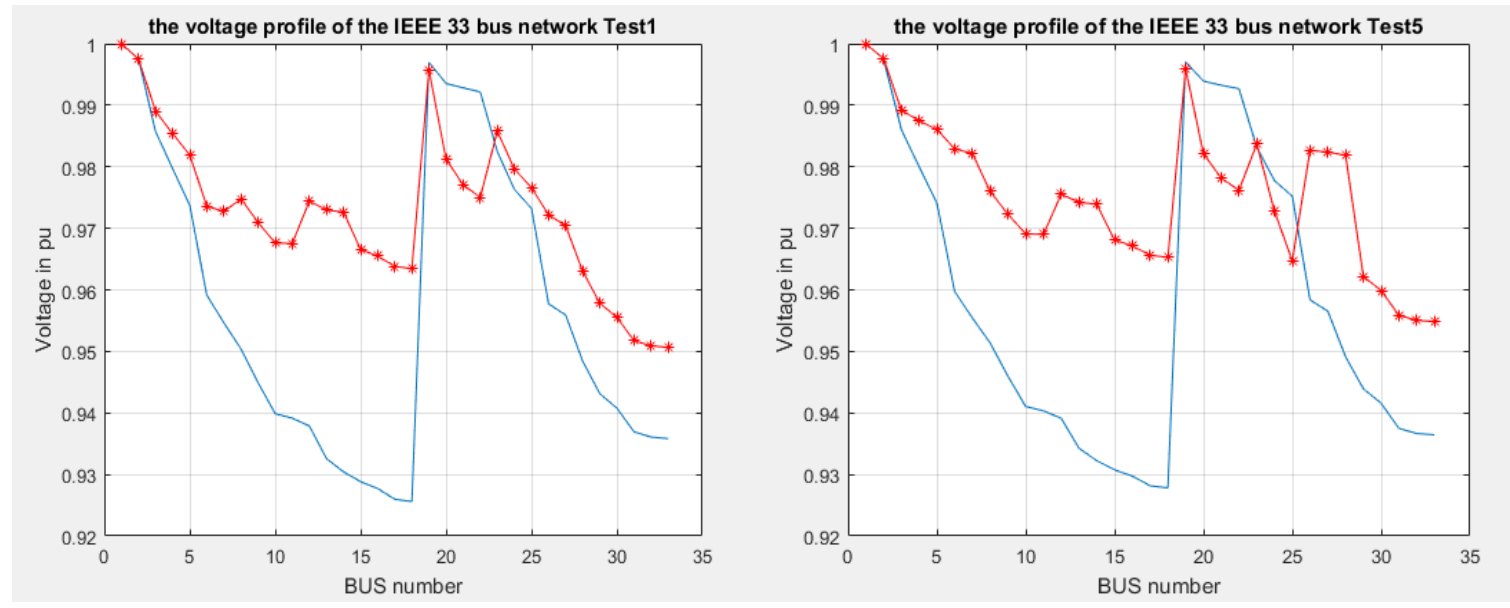

Figure 7. Voltage profile in electrical networks 
All the obtained solutions fully respect the constraints especially the voltage limit. We can say that the reconfiguration of distribution networks is a very powerful tool for managing the electricity distribution networks and the remote implementation of this solution makes them more flexible.

In Figure 9, a comparison based on the power losses is exposed. We can point out that in the all the tests, the reconfiguration helps to reduce the losses. A reduction of $44,45 \%$ is obtained in power losses just by operating this solution. Also, it can be noted that there is a strong link between the branch current which means the power losses and the reliability of the electrical networks. Reducing losses contributes to improving the reliability of electrical networks [33].

All the tests, especially tests 4 and 5 that generate the value randomly, demonstrate the performance of the solution proposed in this work. In the next section, the test will be carried out in real time and validation will be performed using an IEEE33 bus network simulator.

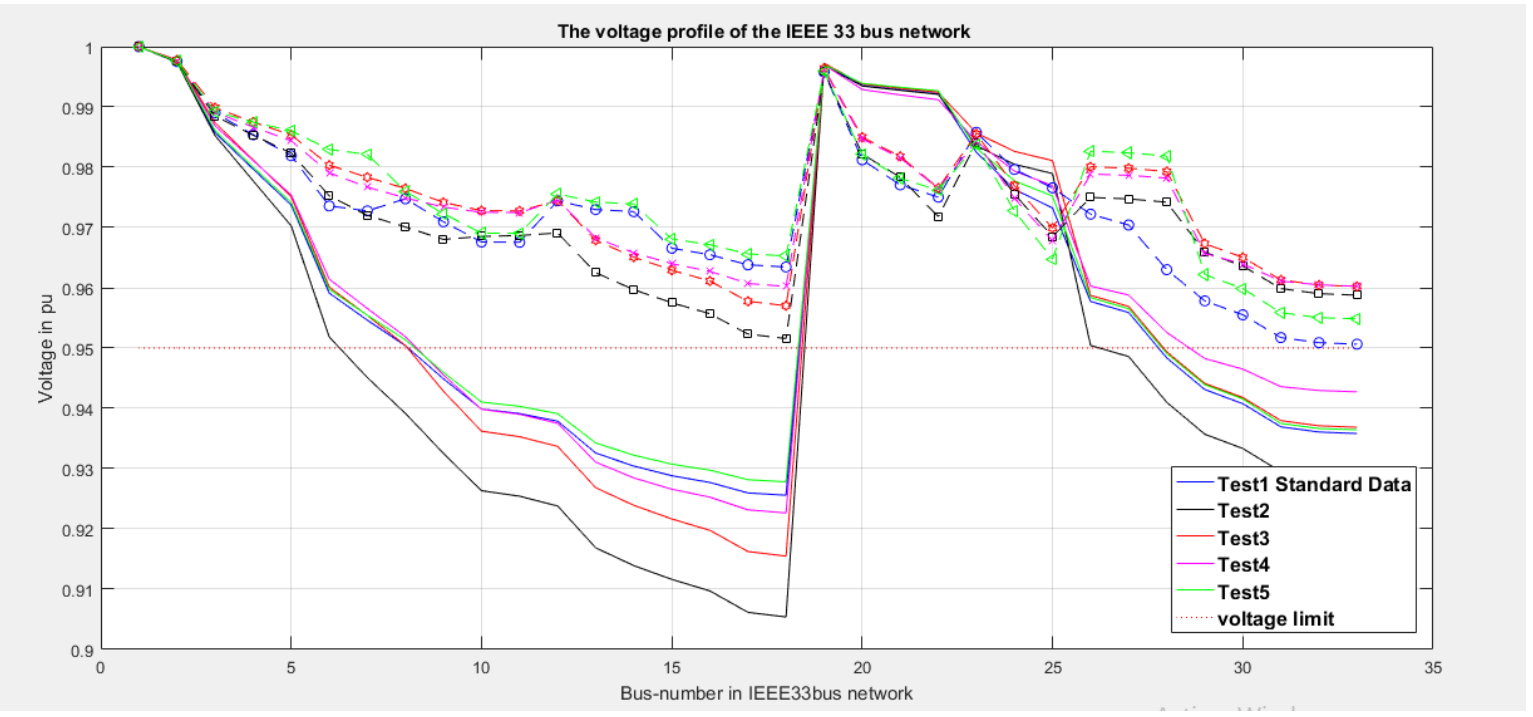

Figure 8. Voltage profile of the IEEE 33 bus for all tests
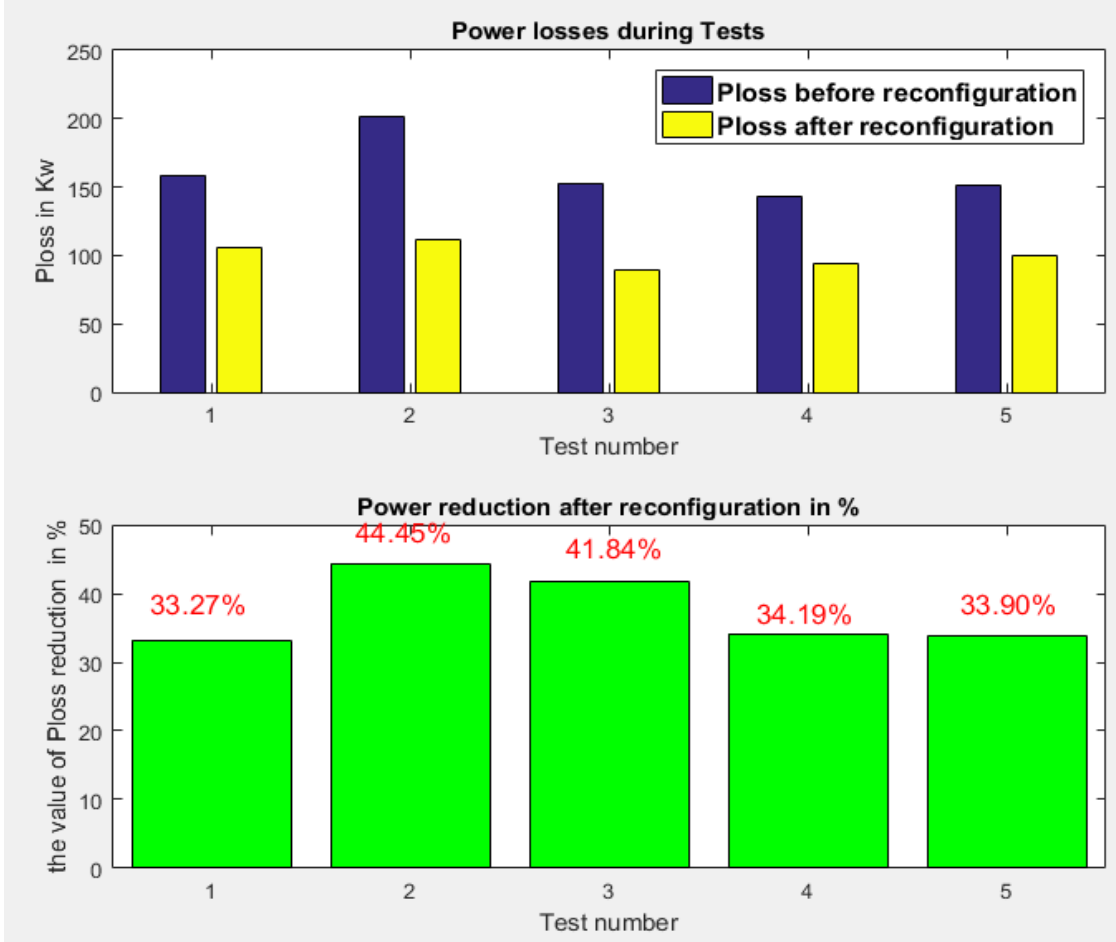

Figure 9. The power losses during all tests 


\section{SOLUTION IMPLEMENTATION AND TEST}

To test the capacity of this solution to control an electrical network in real time, we realized a network emulator using raspberry PI to simulate the operation of the IEEE 33 bus network. The switches are concretized by relays, see Figure 10 . We have changed the voltage and the system generates orders to automatically change the topology.
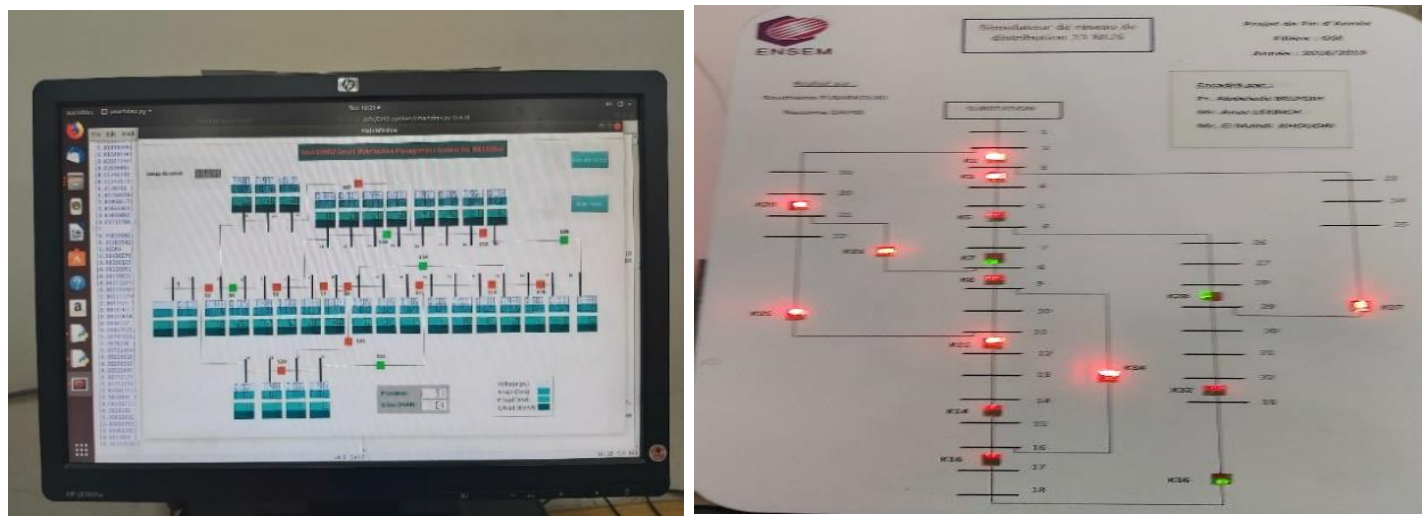

Figure 10. IEEE 33 bus EMULATOR to test the solution

\section{CONCLUSION}

The the reconfiguration of the intelligent electrical distribution networks is converted into multi-criteria optimization problem with real operating constraints to generate the different orders and satisfy the objectives of reliability, safety and cost of electrical energy.

A resolution method is developed to give the system the capacity as well as the intelligence necessary for decision making. thereafter and by exploiting the progress in communication system based on the IoT, the decisions will be sent remotely which will actually contribute to the optimization of intelligent electrical distribution networks. In addition to the improvement of the profile of tension, the load, the most important advantage is the future benifices which can recorded in term of reliability and energy efficiency in particular that the limitation of the current electric networks .

Energy management companies must accelerate the transformation into digital companies by applying powerful software tools to analyze customer needs and meet their requirements. Implement intelligent energy management systems with the capacity to taking the necessary decisions in real time, and the deployment of the IoT in a secure manner to facilitate the interaction between equipment and communication between the management system and customers in order to promote the decentralization of energy production.

\section{REFERENCES}

[1] K. Liu, et al., "Optimal sitting and sizing of DGs in distribution system considering time sequence characteristics of loads and DGs," International Journal of Electrical Power \& Energy Systems, vol. 69, pp. 430-440, 2015, doi: 10.1016/j.ijepes.2015.01.033.

[2] J. Joy , J. DEA, VR. John, "Challenges of Smart Grid," International Journal of Advanced Research in Electrical, Electronics and Instrumentation Engineering (IJAREEIE),vol. 2, pp. 976-81, 2013.

[3] A. Lekbich, A. Belfqih, N. Cherkaoui, F. Elmariami, J. Boukherouaa, O. Sabri, M. N. Dazahra, "A Secure Machine-to-Machine Wireless Communication Using DNP3 Protocol for Feeder Automation in Smart Grid," International Symposium on Ubiquitous Networking UNet, Springer, Cham, pp. 275-286, 2017, doi: 10.1007/9783-319-68179-5_24.

[4] M. Rayati, et al., "An optimal and decentralized transactive energy system for electrical grids with high penetration of renewable energy sources," Electrical Power and Energy Systems, vol. 113, pp. 850-860, 2019, doi: 10.1016/j.ijepes.2019.06.017.

[5] S. Ghasemi, "Balanced and unbalanced distribution networks reconfiguration considering reliability indices," Ain Shams Engineering Journal, vol. 9, no. 4, pp. 1567-1579, December 2018, doi: 10.1016/j.asej.2016.11.010.

[6] P. Zhang, W. Li , Sh.Wang, "Reliability-oriented distribution network reconfiguration considering uncertainties of data by interval analysis," Electrical Power and Energy Systems, vol. 34, no. 1, pp. 138-144, 2012, doi: 10.1016/j.ijepes.2011.08.026. 
[7] D. L. Duan, et al, "Reconfiguration of distribution network for loss reduction and reliability improvement based on an enhanced genetic algorithm," Electrical Power and Energy Systems, vol. 64, pp. 88-95, 2015, doi: 10.1016/j.ijepes.2014.07.036.

[8] D. Shirmohammadi and H. W. Hong, "Reconfiguration of electric distribution networks for resistive line losses reduction," in IEEE Transactions on Power Delivery, vol. 4, no. 2, pp. 1492-1498, April 1989, doi: $10.1109 / 61.25637$

[9] A. A. Mohd Zin, A. K. Ferdavani, A. B. Khairuddin and M. M. Naeini, "Reconfiguration of Radial Electrical Distribution Network Through Minimum-Current Circular-Updating-Mechanism Method," in IEEE Transactions on Power Systems, vol. 27, no. 2, pp. 968-974, May 2012, doi: 10.1109/TPWRS.2011.2174258.

[10] G. Dileep,"A survey on smart grid technologies and applications," Renewable Energy, vol. 146, pp. 2589-2625, February 2020, doi: 10.1016/j.renene.2019.08.092.

[11] S. M. Ali, et al., "Wide area smart grid architectural model and control: A survey," Renewable and Sustainable Energy Reviews, vol. 64, pp. 311-328, 2016, doi: 10.1016/j.rser.2016.06.006.

[12] N. Suljanovic, D. Borovina, M. Zajc, J. Smajic and A. Mujcic, "Requirements for communication infrastructure in smart grids," 2014 IEEE International Energy Conference (ENERGYCON), 2014, pp. 1492-1499, doi: 10.1109/ENERGYCON.2014.6850620.

[13] Bharat Menon Radhakrishnan, Dipti Srinivasan, "A multi-agent based distributed energy management scheme for smart grid applications," Energy, vol. 103, pp. 192-204, 2016, doi: 10.1016/j.energy.2016.02.117.

[14] L. Wanga,, R. Yanb,T. K. Sahab, "Voltage regulation challenges with unbalanced PV integration in low voltage distribution systems and the corresponding solution," Applied Energy, vol. 256, 15 December 2019, doi: 10.1016/j.apenergy.2019.113927.

[15] C. Zedak, A. Lekbich, A. Belfqih, J. Boukherouaa, T. Haidi and F. El Mariami, "A proposed secure remote data acquisition architecture of photovoltaic systems based on the Internet of Things," 2018 6th International Conference on Multimedia Computing and Systems (ICMCS), 2018, pp. 1-5, doi: 10.1109/ICMCS.2018.8525902.

[16] A. Lekbich, A. Belfqih, C. Zedak, J. Boukherouaa and F. El Mariami, "A secure wireless control of Remote Terminal Unit using the Internet of Things in smart grids," 2018 6th International Conference on Wireless Networks and Mobile Communications (WINCOM), 2018, pp. 1-6, doi: 10.1109/WINCOM.2018.8629620.

[17] P. Jafary, S. Repo and H. Koivisto, "Security solutions for smart grid feeder automation data communication," 2016 IEEE International Conference on Industrial Technology (ICIT), 2016, pp. 551-557, doi: 10.1109/ICIT.2016.7474809.

[18] E. Tebekaemi, D. Wijesekera, "Secure overlay communication and control model for decentralized autonomous control of smart micro-grids," Sustainable Energy, Grids and Networks, vol. 18, 2019, doi: 10.1016/j.segan.2019.100222.

[19] H. Fallahzadeh, et al., "Decentralized and hierarchical voltage management of renewable energy resources in distribution smart grid," Electrical Power and Energy Systems, vol. 100, pp. 117-128, 2018, doi: 10.1016/j.ijepes.2018.02.006.

[20] A. Marinia, et al., "An efficient graph-based power flow algorithm for electrical distribution systems with a comprehensive modeling of distributed generations," Electric Power Systems Research, vol. 170, pp. 229-243, May 2019, doi: 10.1016/j.epsr.2018.12.026

[21] U. Ghatak, V. Mukherjee, "A fast and efficient load flow technique for unbalanced distribution system," International Journal of Electrical Power \& Energy Systems, vol. 84, pp. 99-110, January 2017, doi: 10.1016/j.ijepes.2016.05.002.

[22] A. Lekbich, A. Belfqih, T. Ouaderhman, J. Boukherouaa and C. Zedak, "A Remote Decentralized Reconfiguration Strategy of Smart Grid using the Internet of Things," 2019 4th World Conference on Complex Systems (WCCS), 2019, pp. 1-6, doi: 10.1109/ICoCS.2019.8930729.

[23] S. M. Mohd Shokri, M. M. Othman, M. A. Abdul Razak, I. Musirin, Z. Zakaria and M. N. Kari, "A direct approach used for solving the distribution system and harmonic load flow solutions," 2013 IEEE 7th International Power Engineering and Optimization Conference (PEOCO), 2013, pp. 708-713, doi: 10.1109/PEOCO.2013.6564638.

[24] C. Zedak, et al., "Optimal planning and management of photovoltaic sources and battery storage systems in the electricity distribution networks," Przegląd Elektrotechniczny, 2020, doi:10.15199/48.2020.08.19

[25] A. Lekbich, et al., "An analytical multicriteria model based on graph theory for reliability enhancement in distribution electrical networks," International Journal of Electrical and Computer Engineering (IJECE), vol. 9, no. 6, pp. 4625-4636, December 2019, doi: 10.11591/ijece.v9i6.pp4625-4636.

[26] C. Zedak, A. Belfqih, A. Lekbich, J. Boukherouaa and A. Laamimi, "Implementation of an Intelligent System for Remote Control of Decentralized photovoltaic Sources using the Internet of Things Infrastructure," 20197 th International Renewable and Sustainable Energy Conference (IRSEC), 2019, pp. 1-6, doi: 10.1109/IRSEC48032.2019.9078247.

[27] Z. Sheng, C. Mahapatra, C. Zhu and V. C. M. Leung, "Recent Advances in Industrial Wireless Sensor Networks Toward Efficient Management in IoT," in IEEE Access, vol. 3, pp. 622-637, 2015, doi: 10.1109/ACCESS.2015.2435000.

[28] M. M. Hasan and H. T. Mouftah, "Optimal Trust System Placement in Smart Grid SCADA Networks," in IEEE Access, vol. 4, pp. 2907-2919, 2016, doi: 10.1109/ACCESS.2016.2564418.

[29] A. Sajid, H. Abbas and K. Saleem, "Cloud-Assisted IoT-Based SCADA Systems Security: A Review of the State of the Art and Future Challenges," in IEEE Access, vol. 4, pp. 1375-1384, 2016, doi: 10.1109/ACCESS.2016.2549047. 
[30] C. W. Potter, A. Archambault and K. Westrick, "Building a smarter smart grid through better renewable energy information," 2009 IEEE/PES Power Systems Conference and Exposition, 2009, pp. 1-5, doi: 10.1109/PSCE.2009.4840110.

[31] R. J. Tom and S. Sankaranarayanan, "IoT based SCADA integrated with Fog for power distribution automation," 2017 12th Iberian Conference on Information Systems and Technologies (CISTI), 2017, pp. 1-4, doi: 10.23919/CISTI.2017.7975732.

[32] H. W. Chen and F. J. Lin, "Converging MQTT Resources in ETSI Standards Based M2M Platform," 2014 IEEE International Conference on Internet of Things (iThings), and IEEE Green Computing and Communications (GreenCom) and IEEE Cyber, Physical and Social Computing (CPSCom), 2014, pp. 292-295, doi: 10.1109/iThings.2014.52.

[33] A. Kavousi-Fard, M-R. A-Zadeh, "Reliability enhancement using optimal distribution feeder reconfiguration," Neurocomputing, vol. 106, pp. 1-11, 15 April 2013, doi: 10.1016/j.neucom.2012.08.033.

\section{BIOGRAPHIES OF AUTHORS}
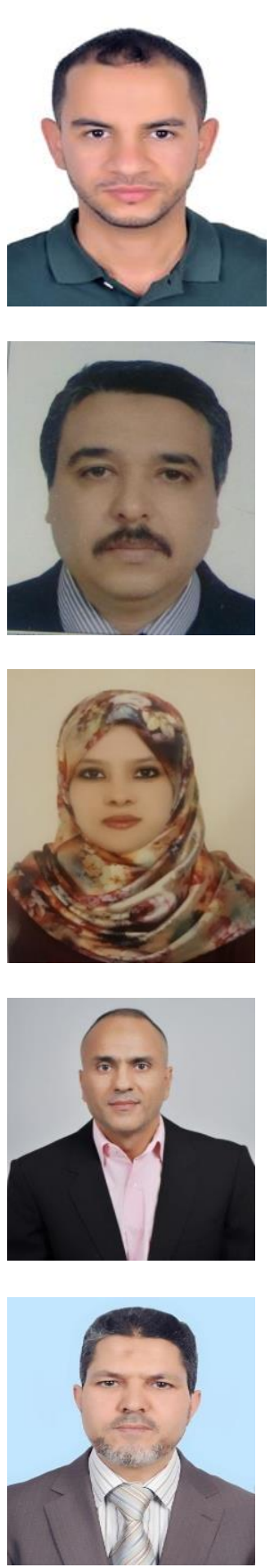

Anass Lekbich: Phd student at the National School of Electricity and Mechanics (ENSEM-Hassan II University of Casablanca), was born in Morocco on the 19th of November 1988. He got his Eng. Degree from ENSEM-Hassan II University of Casablanca, in 2012. He has more than 8 years of professional experience in the industry, as automation and networks engineer with experience in research. He has carried out several research projects on the reliability of the electrical distribution networks, the control of the power systems, the optimization of distribution networks and the location of the protective equipment. His research interests include electrical networks, distribution system, reclosers and switches placement, SCADA system, The remote control of electrical networks and the smart grids.

Pr. Abdelaziz Belfqih: Professor qualified to Direct Research at the National School of Electricity and Mechanics (ENSEM-Hassan II University of Casablanca). Doctor, Engineer and holder of the University Habilitation HDR. RECS Research Team Leader. Currently working on Electrical networks.

Chaimae Zedak: PhD student at the National Higher School of Electricity and Mechanics (ENSEM-Hassan II University of Casablanca). She got his Engineering Degree in electrical engineering, electronics and telecommunications from ENSEM-Hassan II University of Casablanca, in 2016. Her research interests include distribution electrical systems, renewable energies, energy management and remote monitoring and control of electrical networks and renewable sources.

Pr. Jamal Boukherouaa: Professor qualified to Direct Research at the National School of Electricity and Mechanics (ENSEM-Hassan II University of Casablanca). Doctor, Engineer and holder of the University Habilitation HDR. RECS Research Team Leader. Currently working on high-frequency static converters.

Pr. Faissal El Mariami: Professor qualified to Direct Research at the National School of Electricity and Mechanics (ENSEM-Hassan II University of Casablanca). Doctor, Engineer and holder of the University Habilitation HDR. RECS Research Team Leader. Currently working on location of FACTS in Electrical networks. 